\title{
Spiubsaúde
}

\section{A percepção dos professores sobre respiração oronasal em alunos de uma escola da rede privada - São Luís/MA}

\author{
The perception of teachers about oronasal breathing in students of a school of the private network - São \\ Luís/MA
}

\author{
Yasmin Valente Machado* ${ }^{*}$, Patrícia Trinta e Farias Coutinho
}

${ }^{1}$ Universidade Ceuma, São Luís, Maranhão, Brasil. *Autor para correspondência. E-mail: yasminvalente54@hotmail.com

\begin{abstract}
Resumo: Introdução: A respiração é indispensável ao funcionamento do organismo, e sua primeira via é o nariz, que interfere diretamente na proteção das vias respiratórias e estimulação do desenvolvimento craniofacial, porém nem todos conseguem usá-lo de forma adequada, o que traz alterações na vida de um indivíduo. Objetivo: A pesquisa teve como objetivo identificar a percepção dos professores sobre respiração oronasal em alunos. Material e Métodos: A pesquisa foi caracterizada como um estudo analítico, observacional, do tipo transversal, e a amostra inicial foi composta por 10 professores do Colégio Co-Educar, independente de idade e tempo de profissão, mas que ensinassem para alunos na faixa etária de 1,5 a 11 anos de idade. Resultados: Os resultados do estudo indicaram que 60\% dos professores possuíam percepção inadequada e 40\% estavam adequados. Discussão: Balthazar et al. (2016) confirmou que a maioria dos professores não percebe adequadamente a respiração oronasal em alunos, pois $70 \%$ deles relataram não perceber o tipo de respiração de seus alunos. Conclusão: Foi constatada a falta de percepção adequada dos professores sobre respiração oronasal em alunos, e faz-se necessária a criação de atividades que propiciem a conscientização sobre o modo respiratório incorreto e suas consequências, pois os professores podem perceber com mais facilidade não só as alterações físicas, como principalmente quaisquer possíveis interferências delas na aprendizagem.
\end{abstract}

Palavras-chave: Fonoaudiologia, Respiração, Estudantes.

\begin{abstract}
Introduction: The breathing is indispensable to the functioning of the organism, and its first route is the nose, which directly interferes in the protection of the respiratory tract and the stimulation of the craniofacial development, however not everyone can use it properly which brings about changes in the life of an individual. Objective: The research aimed to identify the teachers' perception of oronasal breathing in students. Material and methods: The research was characterized as an observational, cross-sectional analytical study, and the initial sample was composed of 10 Co-Educar school teachers, regardless of age and time of profession, but who taught for students in the age group 1, 5 to 11 years old. Results: The results of the study indicated that $60 \%$ of teachers had inadequate perception and $40 \%$ were adequate. Discussion: Balthazar et al. (2016) confirmed that most teachers do not adequately perceive oronasal breathing in students, as $70 \%$ of them reported not perceiving their students' breathing type. Conclusion: It was verified the teachers' lack of adequate perception about oronasal breathing in students, and it demonstrates the necessity of the creation of activities that promote awareness about the incorrect way of breathing and its consequences, since teachers can perceive more easily not only the physical alterations, as well as any potential interference in the process of learning.
\end{abstract}

Keywords: Speech therapy, Breathing, Students.

\section{Introdução}

A respiração está incluída nos fatores essenciais da função vital nutritiva, juntamente com a circulação, alimentação, excreção, e como reconheceram Lowe e Anderson (2015), é um processo fisiometabólico que permite captar o oxigênio do ambiente para transportá-lo ao sangue, onde ocorre a distribuição corporal celular.

A respiração normal é feita por via nasal, permitindo que o ar inspirado pelo nariz seja purificado, filtrado, aquecido e umidificado antes de chegar aos pulmões. Este modo respiratório é protetor das vias aéreas superiores e também é responsável pelo desenvolvimento adequado do complexo craniofacial (Bianchini et al., 2007).

Alguns indivíduos porém não conseguem realizar o arquétipo respiratório correto, tanto por circunstâncias obstrutivas ou viciosas, o que gera maior risco de doenças, conforme Guyton e Hall (2006) definiram, devido ao fato de que a boca não possui o mecanismo importante para a filtração de partículas, além de alterações antes impensáveis, algumas descritas por Burger et al. (2004) e Balthazar et al. (2016), como cansaço, déficit de atenção, concentração e memória. 
Uma "simples mudança de hábito", como a respiração oronasal é discriminada popularmente, vem acarretando consideráveis problemas no público pediátrico, que não corrigidos durante a infância, promovem agravos sérios até a vida adulta, suscitando o desenvolvimento anormal dentocraniofacial, exemplificado por Guimarães (2013) em desarmonias oclusais, atresias maxilares, faces alongadas, e também no músculo, como bochechas flácidas ou línguas interpostas. Todas estas mudanças interferem nas funções estomatognáticas, favorecendo uma fala imprecisa, mastigação incoordenada, deglutição ruidosa, e podem até mesmo estarem relacionadas com hábitos deletérios, como afirmado por Passos e Frias-Bulhosa (2010) e Vasconcelos et al. (2009).

Nesta observação tardia dos efeitos, um só profissional não tem propriedade absoluta de atender à grande extensão da demanda, havendo necessidade da equipe multidisciplinar para reabilitá-lo, e Izuka (2008) define: o fonoaudiólogo, otorrinolaringologista, o cirurgião-dentista, pediatra, fisioterapeuta, alergologista, psicólogo, todos envolvidos nos diversos aspectos referentes às suas relativas áreas de atuação.

Segundo Gampel (2009), a fonoaudiologia deve instruir o paciente sobre prejuízos causados por padrões respiratórios inadequados e trabalhar o restabelecimento da respiração nasal, que mesmo após a remoção dos obstáculos estruturais nem sempre retorna, e chega com frequência acompanhada de outras modificações no sistema sensório motor-oral, ou seja, cabe a este terapeuta reeducar miofuncionalmente, sintonizar estruturas duras e moles, ajustar a coordenação entre respiração, fala, expressão, mastigação e deglutição para um modelo mais normalizado.

Martins (1999) dá ênfase ao tratamento dos hábitos deletérios, especialmente quando associados à respiração oronasal, indicando também a abordagem multidisciplinar como passo necessário para que haja sucesso na terapêutica.

Na atualidade, a respiração oral é um dos problemas mais preocupantes de saúde pública. Dependendo de sua duração, pode provocar diversas alterações. Estas alterações podem trazer consequências danosas para a qualidade de vida do indivíduo devido ao seu impacto pessoal, físico, psicológico e no relacionamento social (Menezes et al., 2006).

Ao descobrir que respiradores oronasais apresentam pior qualidade de vida nos aspectos ambientais, psicológico e físico, Nagae et al. (2013) confirmou portanto, que a relação entre respiração oronasal e saúde não deve ser subestimada, e muito menos ignorada.

Usando como referência a pesquisa de Silva (2005), que avaliou alunos respiradores oronasais e encontrou um histórico de problemas relacionados à escrita, leitura e matemática, chegamos à questão da participação do professor na percepção da respiração oronasal, em que ele convive com as crianças quase tanto como os pais, e têm maior chance de perceber sinais e sintomas que interfiram em seu desempenho escolar, porém não é o que acontece, como Godoy e Kajihara (2008) constataram, já que o nível de conhecimento dos professores do ensino regular e da educação especial não era suficiente para identificar as alterações e nem correlacionar respiração oronasal com dificuldades de aprendizagem.

Quando Pereira (2001) descobriu que as professoras não percebiam de forma satisfatória estudantes que respiravam de boca aberta, e unindo aos dados da avaliação que detectou crianças respiradoras oronasais na sala que tinham dificuldade para se concentrar, ou já tinham reprovações, deduzimos que poucas pessoas têm informações sobre respiração oronasal, hábitos deletérios ou quaisquer consequências. Desta forma, faz-se necessária a nivelação adequada da atenção sobre este desconhecido problema de saúde pública no mundo, e algo desta magnitude só pode acontecer através de um meio: o esclarecimento.

Os impactos produzidos pela falta de conhecimento sobre o assunto, este bem destacado pelo fato das pesquisas estarem quase sempre enfocadas nos pacientes, e não dando a devida atenção aos próximos em convívio deles, agentes que teriam percebido sinais prévios e perderam a chance de serem essenciais na rapidez desse processo, simplesmente pela ausência de um grau mínimo de instrução, chamaram a atenção para esta questão tão inexplorada e relevante. Assim sendo, identificar a percepção dos professores sobre o tema em relação aos estudantes poderia trazer à consciência deles, os sujeitos que conseguiriam acompanhar os prejuízos de concentração e aprendizagem de um aluno respirador oronasal, mais este fator potencial para desatenção, além dos neurológicos e comportamentais comumente apontados, o que ajudaria em ações mais adequadas a este tipo de caso.

O objetivo deste trabalho foi identificar a percepção dos professores sobre respiração oronasal em alunos. Desta forma, a descrição da pesquisa pode ajudar os professores na compreensão da alteração e também suas influências sobre capacidades de aprendizagem, atenção e memória nos estudantes.

\section{Material e métodos}

O estudo se caracteriza como analítico, observacional, do tipo transversal, e conforme parecer 1.900.192/2017, foi avaliado e aprovado pelo Comitê de Ética em Pesquisa do Hospital Universitário da 
Universidade Federal do Maranhão. O público-alvo foi selecionado no colégio de ensino infantil e fundamental Co-Educar, pertencente à rede privada de ensino, localizada em São Luís - MA. A instituição autorizou a realização da pesquisa apenas durante o turno matutino, portanto, todos os professores do período que se encaixavam dentro dos critérios de inclusão foram convidados a participar, porém somente 10 aceitaram, com os outros justificando não possuir tempo apropriado para colaborar com o estudo. Os critérios de inclusão utilizados exigiram professores que ensinassem para alunos na faixa etária de 1,5 a 11 anos de idade, independente de idade e tempo de profissão. Todos que concordaram em participar assinaram um termo de consentimento livre e esclarecido. A coleta de dados ocorreu no ano de 2017.

Um questionário adaptado do estudo de Borges (2014) foi aplicado aos professores, e dividido em duas etapas, uma primeira obrigatória a todos, que se resumia às três primeiras questões de cunho não-específico, mas com potencial para identificar um professor com ausência de percepção sobre respiração oronasal em alunos, e outra destinada aos que conseguiram transpor às perguntas obrigatórias, sendo esta segunda etapa composta de conteúdo específico sobre o tema, apta apenas para os sujeitos que apresentassem uma percepção significativa, ainda que inferior.

Para a análise de dados foram obtidas primeiramente as distribuições absolutas e percentuais para cada item isolado do questionário, e para uma avaliação geral, como instrumento de medida da percepção dos professores sobre respiração oronasal em alunos, foi criada uma pontuação com uso de escores, em que a somatória total dos itens respondidos por cada professor foi organizada em uma escala. Os resultados foram demonstrados por meio de tabelas e gráficos.

\section{Resultados}

A amostra inicial foi constituída por 10 (100\%) professores, dos quais quatro (40\%) lecionavam no ensino infantil e $6(60 \%)$ no ensino fundamental do $1^{\circ}$ ao $4^{\circ}$ ano. Quanto às formações acadêmicas, 9 (90\%) apresentaram graduação em Pedagogia e 1 (10\%) em Letras.

Na Tabela 1 estão os resultados referentes às distribuições absolutas e percentuais dos hábitos deletérios observados pelos professores em alunos. Observam-se que os hábitos mais encontrados foram que 4 usavam chupeta (40\%), 4 chupavam dedo (40\%) e 4 roíam unhas (40\%), seguido de 2 com uso prolongado de mamadeira (20\%) e 1 apertava dentes (10\%).

Tabela 1. Hábitos observados pelos professores em alunos ( $\mathrm{A}=10)$.

\begin{tabular}{ccc}
\hline Hábitos observados & $\mathrm{N}$ & $\%$ \\
\hline Chupeta & 4 & $40 \%$ \\
Chupar Dedo & 4 & $40 \%$ \\
Ainda Usa Mamadeira & 2 & $20 \%$ \\
Roer Unhas & 4 & $40 \%$ \\
Apertar Dentes & 1 & $10 \%$ \\
\hline
\end{tabular}

Em relação à possibilidade de respirar pela boca trazer algum problema (Figura 1), 6 (60\%) afirmaram que sim e 4 (40\%) não.

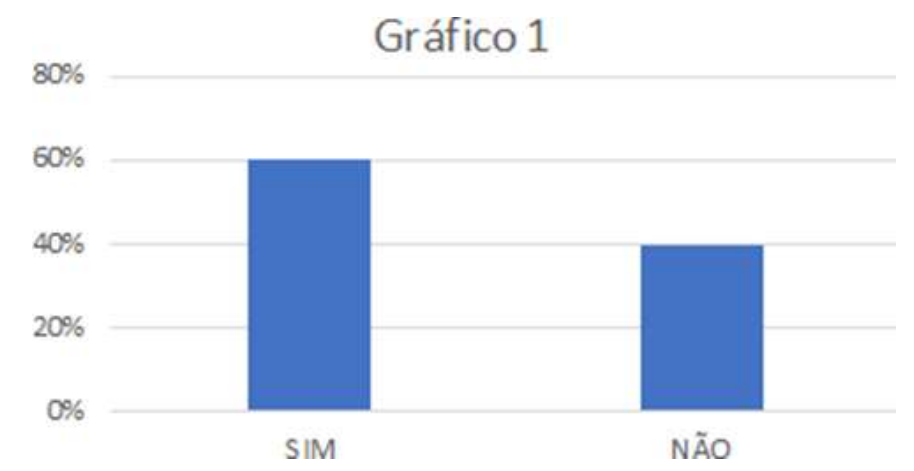

Figura 1. Os professores acham que respirar pela boca traz problemas ou não $(A=10)$.

Quanto ao modo adequado de respirar, obteve-se que $6(60 \%)$ consideraram a respiração oronasal como padrão e $4(40 \%)$ consideraram a nasal. Importante destacar que todos os que consideraram não existir problema em respirar pela boca também optaram pelo modo de respiração oronasal, havendo relação significativa entre as variáveis. 
Valendo-se do parâmetro estabelecido em que aqueles que não considerassem uma participação oral na respiração causadora de prejuízos estariam inaptos a responder a segunda etapa do questionário, cujo conteúdo possuía principalmente perguntas sobre possíveis consequências negativas do modo inadequado de respiração, quatro professores não prosseguiram às outras questões.

De acordo com o critério citado acima, já considerando a partir deste ponto apenas os 6 professores restantes como o total da amostra usada na segunda etapa do questionário (100\%), em relação aos sinais e sintomas que eles achavam serem apresentados por um indivíduo que utiliza a boca para respirar, observouse que 5 optaram pelo ronco (83,3\%), seguido de boca aberta com 4 (66,7\%), ser agitado com $4(66,7 \%)$, acordar com baba também com 4 (66,7\%), e 3 com ser ansioso (50\%). Os resultados estão representados na Tabela 2.

Tabela 2. Sinais e sintomas que os professores achavam serem apresentados por um indivíduo que utiliza a boca para respirar (A=6).

\begin{tabular}{ccc}
\hline Sinais e sintomas & $\mathrm{N}$ & $\%$ \\
\hline Boca aberta & 4 & $66,7 \%$ \\
Espirros e entupimento nasal & 2 & $33,3 \%$ \\
Come lento & 2 & $33,3 \%$ \\
É obeso & $16,7 \%$ \\
É agitado & 4 & $66,7 \%$ \\
É ansioso & 3 & $50 \%$ \\
Sono Agitado & 1 & $16,7 \%$ \\
Acorda com Baba & 4 & $36,7 \%$ \\
Come rápido & $4 \%$ \\
É Magro & 2 & $16,7 \%$ \\
Sono Calmo & 1 & $16,7 \%$ \\
Ronca & 1 & $53,3 \%$ \\
\hline
\end{tabular}

Interligado ainda aos mesmos sinais e sintomas relacionados acima, 4 (66,7\%) professores relataram já ter observado duas ou mais destas características em alunos, e 2 (33,3\%) não observaram.

Quanto à possibilidade dos professores que perceberam dois ou mais sinais/sintomas em alunos terem notado também fatores negativos que possam ter interferido nos desempenhos escolares, dos 4 professores que responderam sim à questão anterior, todos (100\%) observaram cansaço e somente 1 (25\%) notou sono.

$\mathrm{Na}$ Tabela 3 estão os profissionais pensados pelos professores para reabilitar um indivíduo que utiliza a boca para respirar. Fonoaudiólogo e otorrinolaringologista, ambos com 4 (66,7\%) foram mais votados, 2 com cirurgião-dentista (33,3\%) e 1 com pediatra (16,7\%).

Tabela 3. Profissionais pensados pelos professores para reabilitar um indivíduo que utiliza a boca para respirar ( $\mathrm{A}=6)$.

\begin{tabular}{cll}
\hline Profissionais & $\mathrm{N}$ & $\%$ \\
\hline Pediatra & 1 & $16,7 \%$ \\
Fonoaudiólogo & 4 & $66,7 \%$ \\
Otorrinolaringologista & 4 & $66,7 \%$ \\
Cirurgião-Dentista & 2 & $33,3 \%$ \\
\hline
\end{tabular}

Em relação à análise geral dos dados obtidos de todos os professores participantes do estudo, uma pontuação de escores foi projetada para obter o resultado final, concluir se os professores percebiam adequadamente ou não a respiração oronasal em alunos. Uma boa percepção foi indicada para valores acima de 50, razoável entre 41 e 49, insatisfatória de 22 a 40, e a ausência total se caracteriza pela presença de respostas apenas nas três primeiras perguntas, independente de escore. Cabe destacar que tanto a ausência como a percepção insatisfatória são considerados inadequados. Na Tabela 4, os escores e níveis de percepção correspondentes foram distribuídos de acordo com cada professor observado.

Tabela 4. Escores gerais e níveis de percepção dos professores (A=10).

\begin{tabular}{ccc}
\hline Professores & Escores & Nível de Percepção \\
\hline 1 & 15 & Ausente \\
2 & 5 & Ausente \\
3 & 5 & Ausente \\
4 & 5 & Ausente \\
5 & 25 & Insatisfatória \\
6 & 40 & Insatisfatória \\
7 & 44 & Razoável \\
8 & 47 & Razoável \\
9 & 51 & Boa \\
10 & 61 & Boa \\
\hline \hline
\end{tabular}


A média de pontuação dos professores se apresentou em 29,8, com valor mínimo em 5 e máximo 61 . Quanto aos percentuais dos níveis de percepção, observou-se 4 (40\%) professores com ausência, 2 (20\%) com nível insatisfatório, 2 (20\%) obtiveram resultados razoáveis e 2 (20\%) apresentaram boa percepção, portanto, conforme os parâmetros estabelecidos, a soma dos ausentes e insatisfatórios forneceu que a maioria dos professores, 6 (60\%) possuíam percepção inadequada sobre respiração oronasal em alunos e 4 (40\%) estavam adequados.

\section{Discussão}

O nível de percepção dos professores sobre respiração oronasal em alunos é um assunto controverso, pois há uma carência de trabalhos na literatura focados nesta direção. Presume-se que para um professor perceber um modo respiratório inadequado, deve ser necessário o mínimo de entendimento sobre o tema e aplicá-lo ao observar os alunos, critério adotado no presente trabalho.

A respeito da presença de hábitos bucais deletérios infantis, Vasconcelos et al. (2009) estudou uma população de crianças entre 5 a 12 anos de idade, e descobriu que a maioria possuía o hábito de onicofagia (44,6\%), seguido pelo bruxismo (12,6\%), sucção digital $(9,7 \%)$ e sucção de chupeta $(7,4 \%)$, resultados que comparados aos desta pesquisa, confirmaram que roer unhas (40\%) também era um dos hábitos mais observados pelos professores em alunos. Todavia, enquanto chupar dedo e chupeta foram os menos notados em crianças no estudo do outro autor, neste os professores os observaram com a mesma frequência de roer unhas.

Segundo observado no estudo de Passos e Frias-Bulhosa (2010), os hábitos deletérios e o modo respiratório inadequado compartilham uma relação bilateral, pois ambos podem se caracterizar como causa ou consequência um do outro, dependendo da situação apresentada.

Os resultados do estudo de Neto et al. (2009) apontaram que os primeiros casos de respiração bucal da amostra coincidiam com a conservação da chupeta e aumento do uso de mamadeira, o que pode indicar uma possível relação. $O$ autor destaca os 16 primeiros meses de vida como importantes para a instalação do modo respiratório e que as alterações iniciadas neste período costumam passar despercebidas por todas as pessoas ao redor da criança, chamando a atenção para o fato dessas consequências serem percebidas somente com o decorrer dos anos, quando causam distúrbios miofuncionais.

Em relação à possibilidade de respirar pela boca trazer algum problema, este estudo apontou que $60 \%$ dos professores acreditam ser possível e $40 \%$ não, resultado que comparado aos percentuais de $50 \%$ para sim e $50 \%$ para não obtidos por Balthazar et al. (2016), confirmam que apesar de uma parcela significativa dos professores entender que o mecanismo saudável da respiração deve ser realizado pelo nariz, uma parcela significativa também não sabe.

Outra confirmação relevante também foi encontrada, pois quando questionados diretamente quanto ao que pensavam ser o modo adequado de respirar, obteve-se exatamente o mesmo índice nas duas pesquisas, $60 \%$ dos professores optaram pela respiração oronasal e $40 \%$ a nasal. Através desses dois resultados, pode-se supor que os professores ao mesmo tempo em que consideram que respirar pela boca traz prejuízos, não excluem completamente a participação oral nesta função biológica.

Quanto aos sinais e sintomas que os professores achavam ter um indivíduo que utiliza a boca para respirar, os cinco mais frequentes foram ronco (83,3\%), seguido de boca aberta (66,7\%), ser agitado (66,7\%), acordar com baba (66,7\%), e ser ansioso (50\%), resultados compatíveis em sua maioria com os de Borges (2014), cujo os cinco mais encontrados foram ronco $(96,7 \%)$, sono agitado $(93,5 \%)$, ser ansioso $(93,5 \%)$, boca aberta $(93,5 \%)$ e acordar com baba $(87,1 \%)$.

Abreu et al. (2008) também estudou um grupo de 204 crianças respiradoras orais entre 3 a 9 anos de idade e descobriu que os sinais/sintomas mais frequentes foram dormir com a boca aberta (86\%), roncar (79\%), coçar o nariz $(77 \%)$ e babar no travesseiro (62\%), manifestações em grande parte coincididas com as opinadas pelos professores neste estudo, o que pode demonstrar um grau de conhecimento factual sobre esta questão específica.

$\mathrm{Na}$ ausência de trabalhos que trouxessem dados mais exatos sobre fatores que interferem nos desempenhos escolares de possíveis alunos com modo respiratório inadequado, comparamos os resultados obtidos, nos quais todos os professores respondentes do quesito em particular observaram cansaço (100\%) e somente 1 (25\%) notou o sono prejudicando a aprendizagem destes alunos, com os fornecidos por Silva (2005), em que mais da metade de um grupo de 30 crianças com histórico de rinite alérgica e provável respiração oronasal $(54,17 \%)$ foi descrito como tendo problemas de atenção na escola. Os dados das duas pesquisas se complementam, pois, segundo Chedid et al. (2004), a sonolência diurna pode deixar o sujeito desgastado, trazendo prejuízos na codificação, acúmulo e recuperação de informação, consequências passíveis de 
agravamento por comportamentos citados no estudo de Sagaz (2014) como irritação, inquietude e ansiedade, causados devido à uma má oxigenação cerebral.

Borges (2014) também pesquisou quais profissionais os professores recorreriam para reabilitar um indivíduo que utiliza a boca para respirar, e obteve o mesmo índice de $87,1 \%$ para o otorrinolaringologista e cirurgião-dentista, dados não-confirmados inteiramente neste estudo, que encontrou otorrinolaringologista (66,7\%) e fonoaudiólogo (66,7\%).

Finalmente, realizando estudo comparativo quanto aos percentuais encontrados dos níveis de percepção, em que $60 \%$ dos professores possuíam percepção inadequada sobre respiração oronasal em alunos e $40 \%$ estavam adequados, com o índice observado por Balthazar et al. (2016), em que 70\% dos professores relataram não perceber o tipo de respiração de seus alunos, confirma-se que a maioria não percebe adequadamente a respiração oronasal em alunos.

\section{Conclusão}

Pela análise dos dados obtidos pôde-se concluir a falta de percepção adequada dos professores sobre respiração oronasal em alunos, e através deste estudo foi averiguado que faz-se necessária a criação de atividades que propiciem a conscientização sobre o modo respiratório incorreto e suas consequências, pois os professores são personagens que conhecem grande parte do dia-a-dia das crianças e podem perceber com mais facilidade não só as alterações físicas, como principalmente quaisquer possíveis interferências delas na aprendizagem.

Estudos adicionais são necessários, utilizando-se uma amostra maior de professores para investigar a percepção deles sobre o tema em planos mais abrangentes, uma vez que isto também contribui no aumento de informações disponibilizadas ao público.

\section{Referências}

Abreu, R. R., Rocha, R. L., Lamounier, J. A., \& Guerra A. F. 2008. Etiologia, manifestações clínicas e alterações presentes nas crianças respiradoras orais. Jornal de Pediatria, 84(6): 529-35.

Balthazar, I. M., Mariano, N., Nascimento, T. S. B., \& Villena, L. N. 2016. Respiração e Aprendizagem: O que os professores sabem sobre o assunto?. In III Congresso Nacional de Educação. Natal, BR. Natal: Centro Multidisciplinar de Estudos e Pesquisas.

Bianchini, A. P., Guedes, Z. C. F., \& Vieira, M. M. 2007. Estudo da relação entre a respiração oral e o tipo facial. Revista Brasileira de Otorrinolaringologia, 73(4): 500-5.

Borges, E. R. S. 2014. A Percepção de pais e de educadores sobre a respiração bucal. Dissertação de mestrado, Universidade Federal de Alfenas, Alfenas, MG, Brasil.

Burger, R. C. P., Caixeta, E. C., \& Ninno, C. Q. M. S. 2004. A relação entre apnéia do sono, ronco e respiração oral. Revista CEFAC, 6(3): 266-71.

Chedid, K. A. K., Francesco, R. C., \& Junqueira, P. A. S. 2004. A influência da respiração oral no processo de aprendizagem da leitura e escrita em crianças pré-escolares. Revista de Psicopedagogia, 21(65), 157-63.

Gampel, D. 2009. O Respirador Bucal na clínica fonoaudiológica. In T. Sih, A. Chinski, R. Eavey, \& R. Godinho. VIII Manual de Otorrinolaringologia Pediátrica da IAPO (1a ed.). São Paulo, SP: Vida e Consciência.

Godoy, M. A. B., \& Kajihara, O. T. 2008. Formação de professores: nível de conhecimento dos docentes sobre respiração oral. In VIII Congresso Nacional de Educação. Curitiba, BR. Curitiba: Pontifícia Universidade Católica.

Guimarães, S. P. A 2013. Prevalência da respiração bucal e má oclusão em escolares e impacto na qualidade de vida relacionada à saúde bucal. Tese de doutorado, Universidade Federal de Minas Gerais, Belo Horizonte, MG, Brasil.

Guyton, A. C., \& Hall, J. E. 2006. Tratado de Fisiologia Médica (11a ed.). Rio de Janeiro, RJ: Elsevier.

Izuka, E. N. 2008. A influência da respiração oral na oclusão dentária: uma visão geral da literatura. ACTA ORL/Técnicas em Otorrinolaringologia, 26(3): 151-54.

Lowe, J. S., \& Anderson, P. G. 2015. Stevens \& Lowe's Human Histology (4a ed.). Philadelphia: Elsevier.

Martins, B. K. 1999. Respiração Bucal: Uma abordagem multidisciplinar. Monografia de especialização, Universidade Federal de Santa Catarina, Florianópolis, SC, Brasil.

Menezes, V. A., Leal, R. B., Pessoa, R. S., \& Pontes, R. M. E. S. 2006. Prevalência e fatores associados à respiração oral em escolares participantes do projeto Santo Amaro-Recife. Revista Brasileira de Otorrinolaringologia, 72(3): 394-99. 
Nagae, M. H., Alves, M. C., Kinoshita, R. L., Bittencourt, Z. L. C., \& Gagliardo, H. 2013. Qualidade de vida em sujeitos respiradores orais e oronasais. Revista CEFAC, 15(1): 105-10.

Neto, E. T. S., Barbosa, R. W., Oliveira, A. E., \& Zandonade, E. 2009. Fatores associados ao surgimento da respiração bucal nos primeiros meses do desenvolvimento infantil. Revista Brasileira de Crescimento e Desenvolvimento Humano, 19(2): 237-48.

Passos, M. M., \& Frias-Bulhosa, J. 2010. Hábitos de Sucção Não Nutritivos, Respiração Bucal, Deglutição Atípica - Impactos na Oclusão Dentária. Revista Portuguesa de Estomatologia, Medicina Dentária e Cirurgia Maxilofacial, 51(2): 121-27.

Pereira, S. L. 2001. Respiração bucal e Aprendizagem: uma análise fonoaudiológica sobre as causas e consequências. Monografia de especialização, Centro de Especialização em Fonoaudiologia Clínica Motricidade Oral, Itajaí, SC, Brasil.

Sagaz, K. R. 2014. Respiração Oral e Qualidade de Vida: Revisão Bibliográfica. Monografia de especialização, Universidade Federal de Santa Catarina, Florianópolis, SC, Brasil.

Silva, M. D. S. 2005. Problemas de aprendizagem em escolares com rinite alérgica. Dissertação de mestrado, Universidade Estadual de Marin, Maringá, PR, Brasil.

Vasconcelos, F. M. N., Massoni, A. C. L. T., Ferreira, A. M. B., Katz, C. R. T., \& Rosenblat, A. 2009. Ocorrência de Hábitos Bucais Deletérios em Crianças da Região Metropolitana do Recife, Pernambuco, Brasil. Pesquisa Brasileira em Odontopediatria e Clínica Integrada, 9(3): 327-32.

\section{Minicurrículos}

Yasmin Valente Machado. Graduada em Fonoaudiologia pela Universidade Ceuma. Especialista em Atenção em Clínicas Médica e Cirúrgica pelo HU-UFMA.

Patrícia Trinta e Farias Coutinho. Mestre em Fonoaudiologia Clínica pela Pontifícia Universidade Católica PUCSP, Especialista em Disfagia pelo Conselho Federal de Fonoaudiologia - CFFa e Especialista em Motricidade Oral: atuação hospitalar com ênfase em disfagia pelo CEFAC - SP. Atualmente é docente em Fonoaudiologia pela Universidade Ceuma.

Como citar: Machado, Y.V., \& Coutinho, P.T.F. 2020. A percepção dos professores sobre respiração oronasal em alunos de uma escola da rede privada - São Luís/MA. Pubsaúde, 3, a025. DOI: https:// dx.doi.org/10.31533/pubsaude3.a025

Recebido: 7 mar. 2020.

Revisado e aceito: 18 mar. 2020.

Conflito de interesse: os autores declaram, em relação aos produtos e companhias descritos nesse artigo, não ter interesses associativos, comerciais, de propriedade ou financeiros que representem conflito de interesse.

Licenciamento: Este artigo é publicado na modalidade Acesso Aberto sob a licença Creative Commons Atribuição 4.0 (CC-BY 4.0). 


\section{APÊNDICE A}

\section{QUESTIONÁRIO}

Nome:

Formação:

Séries para as quais ensinam:

1 - Vocêjá observou ou tem conhecimento de algum desses hábitos em seus alunos? (MÁXIMO 3 ALTERNATIVAS)
( ) Chupeta
() Chupar dedo
() Ainda usa mamadeira
( ) Roer unhas
( ) Apertar dentes

2 - Na sua opinião, qual o modo mais adequado de respirar?
( ) Nariz e boca
( ) Nariz
( ) Boca

3 - Você acha que respirar pela boca traz algum problema?

( ) Sim ( ) Não

ATENÇÃO: Continue respondendo o questionário apenas se respondeu sim.

\begin{tabular}{|c|c|c|}
\hline $\begin{array}{l}4 \text { - Quais sinais e sintomas você acha que } \\
\text { um indivíduo que utiliza a boca para } \\
\text { respirar tem? (MÁXIMO } 8 \\
\text { ALTERNATIVAS) }\end{array}$ & ( ) Olheiras & ( ) Rosto Longo \\
\hline & ( ) Come Lento & ( ) Come Rápido \\
\hline () Boca Aberta & ( ) É Obeso & () É Magro \\
\hline ( ) Nariz Estreito & ( ) É Agitado & ( ) É Atencioso \\
\hline ( ) Espirros e entupimento & ( ) É Ansioso & ( ) É Calmo \\
\hline nasal frequentes & & \\
\hline ( ) Postura Curva & ( ) Sono Agitado & ( ) Sono Calmo \\
\hline ( ) Cabeça Reclinada a & ( ) Acorda com “Baba” & ( ) Ronca \\
\hline Frente & & \\
\hline
\end{tabular}

5 - Você já observou algum aluno com duas características citadas acima ou mais? ( ) Sim

( ) Não

6 - Caso tenha respondido SIM para a pergunta anterior, notou algum destes fatores interferindo em seu desempenho escolar? (MÁXIMO 2 ALTERNATIVAS)

( ) Sono ( ) Cansaço ( ) Desatenção excessiva ( ) Dificuldade de memorização ( ) NDA

\begin{tabular}{l|l|l|l}
7 - Quais profissionais procuraria para & ( ) Pediatra & ( ) Otorrinolaringologista \\
reabilitar um indivíduo que utiliza a boca \\
para respirar? (MÁXIMO
\end{tabular} \mid $\begin{aligned} & \text { ALTERNATIVAS) } \\
& \text { ( ) Fonoaudiólogo } \\
& \text { ( ) Fisioterapeuta }\end{aligned}$




\author{
APENNDICE B \\ PONTUAÇÃO (ESCORE) - QUESTÕES DE MÚLTIPLAS ALTERNATIVAS \\ 1 - Cada alternativa vale 5 pontos. \\ 4 - Até 3 alternativas assinaladas, cada item vale 2 pontos, e a partir de 4 alternativas marcadas, cada item só vale 1 ponto, com exceção \\ de 3 opções que sempre valerão 0 pontos - É atencioso; é calmo; sono calmo. \\ 6 - Cada alternativa vale 5 pontos, com exceção de NDA (vale 0 pontos). \\ 7 - As alternativas valem: Pediatra - 2 pontos; Otorrinolaringologista - 5 pontos; Fonoaudiólogo - 5 pontos; Médico Clínico-Geral - \\ 1 ponto; Fisioterapeuta - 1 ponto; Cirurgião- Dentista - 2 pontos. \\ PONTUAÇÃO (ESCORE) - QUESTÕES DE UMA ALTERNATIVA CORRETA \\ 2 - As alternativas valem: Nariz e boca - 0 pontos; nariz - 10 pontos; boca - 0 pontos. \\ 3 - Alternativas: Sim - 10 pontos; Não - 0 pontos. \\ 5 - Alternativas: Sim - 5 pontos; Não - 0 pontos.
}

